\title{
Research Square \\ Orai1 Downregulation Causes Proliferation Reduction and Cell Cycle Arrest via Inactivation of the Ras-NF-KB Signaling Pathway in Osteoblasts
}

\author{
Yunshan Guo (D183869503@qq.com ) \\ Hong Hui Hospital, Xi'an Jiao Tong University \\ Dingjun Hao \\ Hong Hui Hospital, Xi'an Jiao Tong University
}

\section{Research Article}

Keywords: Orai1, Ras, NF-KB, cyclin D1, proliferation, osteoblasts

Posted Date: July 29th, 2021

DOI: https://doi.org/10.21203/rs.3.rs-599821/v2

License: (a) (1) This work is licensed under a Creative Commons Attribution 4.0 International License.

Read Full License

Version of Record: A version of this preprint was published at BMC Musculoskeletal Disorders on April 11th, 2022. See the published version at https://doi.org/10.1186/s12891-022-05311-y. 


\section{Abstract}

Background: The purpose of this study was to determine the role of Orai 1 in the regulation of the proliferation and cell cycle of osteoblasts.

Methods: The expression of Orai1 was inhibited by Orai1 small interfering RNA (siRNA) in MC3T3-E1 cells. Following Orai1 downregulation, cell proliferation and cell cycle were examined. Furthermore, the expression of cyclin D1, cyclin E, CDK4, and CDK6 was analyzed. The activity of the Ras-NF-KB signaling pathway was investigated to identify the role of Orai1 in the regulation of osteoblast proliferation.

Results: Orai1 was successfully downregulated in MC3T3-E1 cells by the Orai1 siRNA transfection ( $p$ $<0.05)$. We found that MC3T3-E1 cell proliferation was decreased, and the cell cycle was arrested by Orai1 downregulation $(p<0.05)$. Additionally, the expression of cyclin D1 was decreased by Orai1 downregulation $(p<0.05)$, as was the activity of the Ras-NF-KB signaling pathway $(p<0.05)$. Orai1 siRNA did not further reduce cell proliferation, the proportion of cells in the $S$ phase, and cyclin D1 expression after chemical blockage of the Ras signaling pathway in MC3T3-E1 cells $(p>0.05)$.

Conclusions: The results reveal that Orai1 downregulation may reduce cyclin D1 expression by inactivating the Ras-NF-KB signaling pathway thus blocking osteoblast proliferation and cell cycle.

\section{Introduction}

Osteoporosis is a common degenerative disease of the skeletal system and seriously affects the health of the elderly. The inhibition of osteoblast proliferation leads to the onset and development of osteoporosis [1-5]. Therefore, a study on the mechanism regulating osteoblast proliferation will lead to a new biological treatment of clinical osteoporosis. The calcium ion is an important secondary messenger of cells and is involved in the regulation of cell proliferation, differentiation, migration, and cytokine secretion [6-7]. For excitable cells, a pathway launched by a voltage-dependent calcium channel located on the plasma membrane is the major cascade that mediates the influx of extracellular calcium into the cytoplasm. A voltage-dependent calcium channel is not present in most nonexcitable cells, and recent research indicates that a pathway triggered by a calcium release-activated calcium (CRAC) channel is the primary cascade that mediates extracellular calcium influx into the cytoplasm of nonexcitatory cells. Orai1 is the key component of a CRAC channel. Orai1 mainly comprises four transeptal segments and is a quaternary transmembrane protein. It is located on the plasma membrane, and its $\mathrm{C}$ and $\mathrm{N}$ termini are both located in the cell. The first segment of Orai1 is rich in proline and arginine, and the third transeptal segment is rich in glutamate and can selectively bind and transport calcium ions. When extracellular stimuli cause depletion of endoplasmic-reticulum calcium, stromal interaction molecule 1 (STIM1), located on the endoplasmic omentum, can induce Orai1 to form a calcium channel that promotes calcium ion influx and activates calcium signaling cascades to complete various cellular physiological functions [8-9]. Previous studies have revealed that Orai1 may be a target for the treatment or prevention of the bone loss caused by osteoclasts in the RAW cell line. Orai1 knockdown reduces SOCE and inhibits 
RANKL-induced osteoclastogenesis of RAW264.7 cells, a murine monocyte/macrophage cell line, by suppressing the induction of NFATC1[10]. Osteoblast differentiation and function are affected by Orai1 deficiency. Deletion of the Orai1 gene can lead to a phenotype of osteoporosis, e.g., decreased bone density in mice, suggesting that Orai1 participates in the pathogenesis of osteoporosis [11-13]. Nonetheless, to the best of our knowledge, there are no reports about Orai1 involvement in the proliferation of osteoblast. Our study indicates that Orai1 downregulation leads to a slowdown of osteoblast proliferation and to cell cycle arrest. The Orai1 downregulation inactivates the Ras-NF-KB signaling pathway to reduce the expression of cyclin D1 thus decreasing the proliferation of osteoblasts. The purpose of this study was to uncover new avenues for the treatment of osteoporosis.

\section{Material And Methods}

\section{Cell culture}

Mouse calvaria osteoblasts, MC3T3-E1, were purchased from the American Type Culture Collection (ATCC) and cultured at $37^{\circ} \mathrm{C}$ in a-Minimum Essential Medium supplemented with $10 \%$ of fetal bovine serum (Gibco, NY, USA). The culture medium was refreshed every three days thereafter.

\section{Gene silencing}

The sense sequence for Orai1 small interfering RNA (siRNA) was 5- CGTGCAC $\forall$ TCTC $\forall C T C G$ - 3. Orai1 siRNA was dissolved in the serum-free a-Minimum Essential Medium with the Lipofectamine 2000 reagent for $20 \mathrm{~min}$. Then, $40 \mathrm{nM}$ Orai1 siRNA (or control siRNA) was added into 6-well plates containing MC3T3-E1 cells. The cells were collected for further analysis after $24 \mathrm{~h}$.

\section{Immunofluorescence analysis}

Approximately $10^{4}$ cells per well were added into a 24-well cell culture plate. After the cells were cultured for $4 \mathrm{~h}$, the cells were fixed with a $4 \%$ methanol-free formaldehyde solution and rinsed with PBS twice. Then the Orai1 antibody (Invitrogen, CA, USA) were diluted 1:500 in Tris-buffered saline and incubated with the cells at $37^{\circ} \mathrm{C}$ for $60 \mathrm{~min}$. To detect Orai1, the cells were incubated for $40 \mathrm{~min}$ with a FITCconjugated IgG antibody (Pierce, Illinois, USA) diluted 1:800 in TBST. Finally, the slides were incubated with 4,6-diamidino-2-phenylindole (DAPI) (Pierce, Illinois, USA) to stain nuclei for 20min. The cells were immediately analyzed under a fluorescence microscope with a standard fluorescein filter set (Olympus, Japan) to examine green fluorescence of Orai1 at $520 \mathrm{~nm}$ and blue fluorescence of DAPI-stained nuclei at $460 \mathrm{~nm}$. Each experiment was carried out in triplicate and repeated three times independently.

\section{Western blot analysis}

The pretreated cells were lysed, and the total protein concentration was determined using the BCA Protein Assay Kit (Pierce, Illinois, USA). Equal quantities of cell lysate samples were mixed with loading buffer and then separated by sodium dodecyl sulphate-polyacrylamide gel electrophoresis in a $10 \%$ gel for 90 
$\min$. After that, the proteins were electrophoretically transferred from the gel onto a polyvinylidene fluoride microporous (PVDF) membrane for $60 \mathrm{~min}$ in a semidry chamber. Anti-mouse Orai1 antibodies and GAPDH antibodies were purchased from Invitrogen (Carlsbad, CA, USA). The following anti-mouse antibodies were purchased from Cell Signaling Technology (Beverly, MA, USA): cyclin D1 antibodies, cyclin E antibodies, Ras antibodies, Ras-GRF antibodies, p65-NF-KB antibodies, and phospho- (p-)p65-NFKB antibodies. Anti-mouse CDK4 and CDK6 antibodies, were purchased from Santa Cruz Biotechnology (Santa Cruz, CA, USA). These primary antibodies were diluted 1:800 to 1:1000 in Tris-buffered saline containing $0.1 \%$ of Tween 20 (TBST) and incubated with the PVDF membranes at $37^{\circ} \mathrm{C}$ for $60 \mathrm{~min}$. To detect the primary antibody, the PVDF membranes were probed for $1 \mathrm{~h}$ with a horseradish peroxidaseconjugated anti-rabbit or anti-goat IgG antibody diluted 1:5000 to 1:10000 in TBST. Immunoreactive protein bands were visualized with the Enhanced Chemiluminescence (ECL) Reagent Kit (Millipore, USA) and documented using a Western-Light Chemiluminescent Detection System (Applied Biosystems, USA). Densitometric analyses of the protein bands were performed in the ImageJ software (version 1.48, National Institutes of Health, USA). All fold changes of band densities were determined with normalization to GAPDH, an endogenous control. Relative protein expression was calculated as relative density of a protein band normalized to the endogenous control. Each experiment was conducted in triplicate and repeated three times independently.

\section{Real-time PCR analysis}

Total RNA was extracted from the pretreated cells using the RNA Extraction Kit (Toyobo, Japan). Then, the total RNA was reverse-transcribed into cDNA by means of the cDNA Reverse Transcription Kit (Invitrogen, USA). The forward primer for Orai1 expression analysis was 5- TAC $\top \forall G \mathbb{C} G C G \mathbb{C} \forall G$ - 3, and the reverse primer was 5-ACTTCCACCATCGCTACCA-3. The forward primer for cyclin D1 expression analysis was 5-TTTCTTTCCAGAGTCATCAAGTGT-3, and the reverse primer was 5TGACTCCAGAAGGGCTTCAA-3. The forward primer for cyclin E expression analysis was 5-

TTTCTGCAGCGTCATCCTC-3, and the reverse primer was 5-TGGAGCTTATAGCTTCGCACA-3. The forward primer for CDK4 expression analysis was 5- TCAGTGGTGCCAGAGATGG-3, and the reverse primer was 5GGAAGGCAGAGATTCGCTTA-3. The forward primer for CDK6 expression analysis was 5GCCCTTACCTCGGTGGTC-3 and the reverse primer was 5-ACAGGGGTGGCATAGCTG-3. Real-time PCR was used to measure mRNA expression levels of Orai1, cyclin D1, cyclin E, CDK4, and CDK6. Real-time PCR was carried out with the SYBR green PCR reagent (Takara, Japan) on a Stratagene M63005P Multiplex Quantitative PCR System (Agilent Technologies, Germany). GAPDH served as an endogenous control. The real-time PCR cycling conditions were as follows: denaturation at $95^{\circ} \mathrm{C}$ for $10 \mathrm{~min} ; 35 \mathrm{cycles}$ of denaturation at $95^{\circ} \mathrm{C}$ for $10 \mathrm{~s}$, annealing at $59^{\circ} \mathrm{C}$ for $15 \mathrm{~s}$, and extension at $72^{\circ} \mathrm{C}$ for $20 \mathrm{~s}$; and final extension at $72^{\circ} \mathrm{C}$ for $15 \mathrm{~min}$. The reaction was terminated by cooling at $4^{\circ} \mathrm{C}$. Each experiment was conducted in triplicate and repeated three times independently.

\section{Cell proliferation assay}


Cell proliferation was analyzed by a 3-(4,5-dimethylthiazol-2-yl)-2,5- diphenyltetrazolium bromide (MTT) assay. Approximately $10^{4}$ cells per well were added into a 96-well cell culture plate. After the cells were cultured for $24,48,64$, or $72 \mathrm{~h}, 50 \mu \mathrm{L}$ of MTT (Sigma-Aldrich, USA) and $150 \mu \mathrm{L}$ of the culture medium were added into each well of the cell culture plate. Then, the cell culture plates were incubated at $37^{\circ} \mathrm{C}$ for $4 \mathrm{~h}$. MTT was dissolved in $200 \mu \mathrm{L}$ of DMSO in each well for $30 \mathrm{~min}$. Finally, the absorbance of each well was measured at $570 \mathrm{~nm}$ on a microplate reader (BioAssay Systems, USA). All fold changes of cell proliferation were normalized according to the following formula: (Sample absorbance - Blank absorbance)/(Control absorbance - Blank absorbance). Each experiment was carried out in triplicate and repeated three times independently.

\section{Cell apoptosis assay}

Apoptosis of osteoblasts was detected using a DeadEnd Fluorometric Tunel System (Promega, USA) that end-labels fragmented DNA from apoptotic cells via modified terminal deoxynucleotidyl transferase dUTP nick end labeling (Tunel) staining. Briefly, the cells were fixed with a $4 \%$ methanol-free formaldehyde solution and rinsed with PBS twice, then the fragmented DNA of the apoptotic cells was end-labeled with fluorescein-12-dUTP, and the slides were incubated with 4,6-diamidino-2-phenylindole (DAPI) (Pierce, Illinois, USA) to stain nuclei. The cells were immediately analyzed under a fluorescence microscope with a standard fluorescein filter set (Olympus, Japan) to examine green fluorescence of apoptotic cells at 520 $\mathrm{nm}$ and blue fluorescence of DAPI-stained nuclei at $460 \mathrm{~nm}$. Each experiment was carried out in triplicate and repeated three times independently.

\section{Cell cycle assay}

The cells were cultured in the serum-free medium for $24 \mathrm{~h}$. Then, the cells were cultivated in the medium with $10 \%$ of FBS. After $24 \mathrm{~h}$, the cells were collected into a centrifuge tube and resuspended in $1 \mathrm{~mL}$ of anhydrous ethanol. The cells were incubated overnight at $4^{\circ} \mathrm{C}$. Next, the cells were collected into a centrifuge tube and resuspended in $500 \mu \mathrm{L}$ of a propidium iodide (PI) solution (Absin, China). The cells were stained in at $4^{\circ} \mathrm{C}$ (in a refrigerator) for $60 \mathrm{~min}$. Finally, the cell cycle was examined by flow cytometry, and the data were analyzed statistically. Each experiment was conducted in triplicate and repeated three times independently.

\section{Statistical analysis}

We employed SPSS 16.0 software to analyze the data statistically. All values are expressed as mean \pm standard error from at least three independent experiments. Multigroup comparisons were analysed using one-way (ANOVA) followed by Tukey's post hoc significance difference tests. Student's t test was used for pairwise comparisons. The results were considered statistically significant when a $p$ value was $<0.05$.

\section{Results}

Expression of Orai1 is inhibited by Orai1 siRNA in MC3T3-E1 cells 
Some studies indicate that the deletion of the Orai1 gene can lead to a phenotype of osteoporosis, e.g., decreased bone density in mice, suggesting that Orai1 is involved in the pathogenesis of osteoporosis [67]. To investigate the role of Orai1 in the onset and development of osteoporosis via the regulation of the proliferation of osteoblasts, Orai1 siRNA was transfected into MC3T3-E1 cells to reduce the expression of Orai1. We transfected a control siRNA as negative control. Immunofluorescence and western blotting were performed to assess Orai1 protein expression, and real-time PCR was carried out to evaluate Orai1 mRNA expression after the Orai1 siRNA transfection. We found that Orai1 protein expression and Orai1 mRNA levels were remarkably decreased by the Orai1 siRNA transfection (Figure 1a, 1b and 1c, $p<0.05$ ). The results indicated that Orai1 siRNA successfully inhibited Orai1 expression in MC3T3-E1 cells.

\section{Orai1 downregulation reduces proliferation of MC3T3-E1 cells and causes cell cycle arrest}

A decrease in the number of osteoblasts occurs during the development of osteoporosis. Therefore, the proliferative ability of osteoblasts plays an important role in the progression of osteoporosis. To test whether Orai1 participates in the regulation of the proliferation of osteoblasts, Orai1 siRNA was utilized to suppress the expression of Orai1 in MC3T3-E1 cells. Then, changes in the proliferation of MC3T3-E1 cells were detected by the MTT assay. We noticed that the proliferation of MC3T3-E1 cells significantly decreased in a time-dependent manner after the Orai1 downregulation ( $p<0.05$, Figure $2 a)$. Apoptosis is another important cause of the reduction in the osteoblast number. Alterations of the apoptosis of MC3T3-E1 cells were assessed by Tunel staining. There were no significant changes in the apoptosis of osteoblasts after the Orai1 downregulation ( $p>0.05$, Figure $2 b$ ). This result confirmed that the Orai 1 knockdown reduced the proliferation of osteoblasts.

The process of cell proliferation depends on the switching of phases of the cell cycle. The $\mathrm{S}$ phase is the DNA synthesis phase, which is an important indicator of the cellular proliferative ability. To determine whether Orai1 is involved in the regulation of osteoblast proliferation, we applied PI staining and flow cytometry to detect cell cycle changes in the osteoblasts after the Orai1 knockdown. The results meant that the percentages of cells at the G0-G1 transition and in the S phase of the cell cycle significantly increased and decreased, respectively, after the Orai1 downregulation ( $<<0.05$, Figure $2 c)$. The above results confirmed that the Orai1 downregulation reduces the entry into the $S$ phase and inhibits the proliferation of osteoblasts, resulting in a decreased number of osteoblasts.

\section{The Orai1 knockdown reduces the expression of cyclin D1 in MC3T3-E1 cells}

The progression of the cell cycle depends on two key types of regulatory molecules, cyclins and cyclindependent kinases (CDKs) [8-9]. To investigate whether Orai1 plays a part in the regulation of the cell cycle in osteoblasts, the expression of cyclin D1, cyclin E, CDK4, and CDK6 was measured by western blotting after the Orai1 downregulation. We found that the expression of cyclin D1 in MC3T3-E1 cells significantly decreased after the Orai1 knockdown ( $<<0.05$, Figure $3 \mathrm{~A})$. There was no significant change in the expression of cyclin E, CDK4, and CDK6 in osteoblasts after the Orai1 downregulation ( $p>0.05$, Figure 3a). Then, the mRNA levels of cyclin D1, cyclin E, CDK4, and CDK6 were evaluated by real-time PCR after the Orai1 downregulation. These results indicated that the mRNA level of cyclin D1 was remarkably 
reduced by the Orai1 downregulation ( $p<0.05$, Figure $3 b)$. There were no significant changes in the mRNA levels of cyclin E, CDK4, and CDK6 in osteoblasts after the Orai1 downregulation ( $p>0.05$, Figure $3 b$ ). These results confirmed that the Orai1 downregulation can significantly reduce the expression of cyclin D1, thereby leading to osteoblast cell cycle arrest and a proliferation reduction.

\section{Orai1 downregulation reduces the proliferation of osteoblasts by inactivating the Ras-NF-KB signaling pathway}

It has been reported that inhibition of the Ras-NF-KB signaling pathway leads to a reduction in osteoblast proliferation [10-12]. Some studies have revealed that calcium ions activate the Ras signaling cascade by directly binding to Ras-GRF [13]. To further clarify the mechanism of Orai1 action in the regulation of osteoblast proliferation, alterations in the amounts of Ras-GRF and p-p65-NF-kB, which are activated forms of Ras and NF-KB, were studied after the Orai1 downregulation. We did not detect any significant change in the amounts of total Ras and p65-NF-KB after the Orai1 downregulation. Nonetheless, we found that the presence of Ras-GRF and p-p65-NF-kB were significantly reduced by the Orai1 downregulation ( $p<0.05$, Figure $4 a)$. The results suggested that Orai1 downregulation can reduce the activation of Ras and NF-KB in MC3T3-E1 cells. To further elucidate whether the Orai1 downregulation can inactivate the Ras-NF-KB signaling pathway to reduce osteoblast proliferation, the Ras signaling pathway inhibitor ARS-853 was applied. The ARS-853 stock solution was diluted to a concentration of 50 $\mathrm{nM}$ in the cell culture medium. DMSO alone was dissolved as negative (vehicle) control. After that, western blotting and cell proliferation and cell cycle assays were performed after incubation with or without the Ras signaling pathway inhibitor ARS-853 (50 nM) for an appropriate period. The expression of $p-p 65-N F-k B$ and cyclin D1 was decreased by the Orai1 downregulation $(p<0.05$, Figure $4 b)$. On the other hand, the addition of ARS-853 reduced the amounts of p-p65-NF-kB and cyclin D1 in the control siRNA transfection groups to the levels comparable to the level in the Orai1 siRNA transfection groups. The Orai1 downregulation did not further reduce the amounts of p-p65-NF-kB and cyclin D1 after the blockage of Ras activity ( $>0.05$, Figure $4 b)$. Furthermore, we noted that Orai1 siRNA significantly reduced cell proliferation in the control group ( $p<0.05$, Figure $4 c)$. Nevertheless, the cell proliferation in control siRNA groups was similar to that in Orai1 siRNA groups after ARS-853 was added. Orai1 siRNA did not further reduce the cell proliferation after the blockage of Ras activity ( $p>0.05$, Figure $4 c)$. Moreover, we demonstrated that Orai1 siRNA significantly diminished the proportion of cells in the $S$ phase in the control group ( $p<0.05$, Figure $4 d$ ). Nonetheless, we found that the proportion of cells in the $S$ phase in control siRNA groups was similar to that in Orai1 siRNA groups after ARS-853 was added. Orai1 siRNA did not further reduce the proportion of cells in the $S$ phase after the blockage of Ras activity ( $p$ $>0.05$, Figure $4 \mathrm{~d}$ ). The above results indicated that the activity of the Ras-NF-KB signaling pathway is required for Orai1-regulated proliferation of MC3T3-E1 cells. These results confirmed that the Orai1 downregulation reduced the proliferation of osteoblasts by inactivating the Ras-NF-KB signaling pathway.

\section{Discussion}


Osteoporosis is a common degenerative disease of the skeletal system and seriously affects the health of the elderly. The inhibition of osteoblast proliferation is an important reason for the onset and development of osteoporosis [1-5]. Therefore, further research on the mechanism regulating osteoblast proliferation should reveal a new biological treatment of clinical osteoporosis.

The highly mineralized bone matrix is formed by osteoblasts, which mediate calcium and phosphate deposition in the type I collagen matrix. The calcium ion is an important secondary messenger of cells and participates in the regulation of cell proliferation, differentiation, migration, and cytokine secretion [6-7]. High concentration of the extracellular calcium ion enhances osteogenesis implemented by cultured human mesenchymal stem cells. Studies suggest that vitamin $D$ is the main regulator of calcium homeostasis and bone mineralization and can promote osteogenic differentiation in vitro [14-17]. Some studies have confirmed that osteogenic characteristics of osteoblasts, such as proliferation, differentiation, and extracellular-matrix calcification, are enhanced significantly when the concentration of calcium ions in extracellular fluid increases [18-20]. Other studies show that in patients with osteoporosis, levels of calcium ions in the extracellular fluid of osteoblasts increase nearly 20 -fold owing to overactivation of osteoclasts. Although the extracellular calcium level is significantly increased, the proliferation of osteoblasts in patients with osteoporosis is still slow [21-23]. Calcium ions must enter the cytoplasm to act as a secondary messenger. Recent research suggests that a CRAC channel is a primary player in the pathway that mediates extracellular calcium influx into the cytoplasm of nonexcitatory cells. Orai1 is the key component of a CRAC channel. Orai 1 is mainly composed of four transeptal segments and is a quaternary transmembrane protein. It is located on the plasma membrane, and its $\mathrm{C}$ and $\mathrm{N}$ termini are both located in the cell. The first segment of Orai1 is rich in proline and arginine, and the third transeptal segment is rich in glutamate, which can selectively bind and transport calcium ions. When extracellular stimuli deplete endoplasmic-reticulum calcium, STIM1 located on the endoplasmic omentum can approach Orai1 and induce it to form a calcium channel that promotes calcium ion influx and activates calcium signaling cascades to complete some cellular physiological functions [8-9]. Previous studies have revealed that Orai1 may be a target for the treatment or prevention of the bone loss caused by osteoclasts in the RAW cell line. Orai1 knockdown reduces SOCE and inhibits RANKL-induced osteoclastogenesis of RAW264.7 cells, a murine monocyte/macrophage cell line, by suppressing the induction of NFATC1[10]. Osteoblast differentiation and function are affected by Orai1 deficiency. Deletion of the Orai1 gene can lead to a phenotype of osteoporosis, e.g., decreased bone density in mice, suggesting that Orai1 participates in the pathogenesis of osteoporosis [11-13]. On the other hand, the effects of Orai1 on osteoblasts and its cumulative influence on the stability of the intraosseous environment have not been fully studied; this situation limits our understanding of Orai1 functions in bone biology. Inhibition of osteoblast proliferation leads to a decrease in the number of osteoblasts, which is an important reason for the onset and development of osteoporosis. To investigate whether Orai1 is involved in the onset and development of osteoporosis by regulating the proliferation of osteoblasts, we transfected Orai1 siRNA into MC3T3-E1 cells. We found that the proliferation of osteoblasts was significantly reduced by the Orai1 downregulation in MC3T3-E1 cells. Moreover, we noted that there were no significant changes in the mineralization by MC3T3-E1 cells and in their 
apoptosis after the Orai1 downregulation. These results confirm that the Orai1 downregulation reduces the proliferation of osteoblasts. The process of cell proliferation depends on the switching of cell cycle phases. The S phase of the cell cycle is the DNA synthesis phase, which is an important indicator of the cellular proliferative ability. In this study, we examined changes in the cell cycle of osteoblasts after the Orai1 downregulation. The results revealed that the proportion of cells at the G0-G1 transition of the cell cycle significantly increased and the proportion of cells in the $S$ phase significantly decreased after the downregulation of Orai1. These results mean that an Orai1 knockdown can reduce the proliferation of osteoblasts, thereby decreasing their number.

The progression of the cell cycle depends on two key types of regulatory molecules, cyclins and CDKs. The latter are multifunctional enzymes that phosphorylate various protein substrates during cell cycle progression, especially when the cell cycle advances from the G1 phase to S phase. Cyclins, including cyclins $\mathrm{D} 1$ and $\mathrm{E}$, can bind to and activate CDK4 and CDK6, playing a key role in the progression of the cell cycle from the $\mathrm{G} 1$ phase to $S$ phase [24-25]. CDK activity is also negatively regulated by cyclindependent kinase inhibitors. P27 is a key member of the cyclin-dependent kinase inhibitor family and binds to the cyclin-CDK2 complex in the nucleus and negatively regulates the cell cycle, thus leading to cell cycle arrest in the $\mathrm{G} 1$ phase [26-27]. We found that protein expression and mRNA levels of cyclin E, CDK4, and CDK6 in osteoblasts are not significantly changed by the Orai1 downregulation, whereas the protein amount and mRNA level of cyclin D1 in osteoblasts are significantly reduced by the Orai1 downregulation. These results suggest that the Orai1 knockdown can reduce the expression of cyclin D1 in osteoblasts and arrest the cell cycle between the G1 phase and S phase, thereby slowing the proliferation of osteoblasts.

It has been demonstrated that the suppression of the Ras-NF-KB signaling pathway slows osteoblast proliferation. Growth factors and other mitotic stimuli can induce the expression of cyclin D1 through the Ras-NF-KB signaling pathway [28-30]. Orai1 is a calcium channel protein that mediates receptordependent calcium ion influx. When calcium ions enter the cytoplasm, they act as a secondary messenger and activate multiple signaling pathways to finely regulate various functions. Research indicates that calcium ions activate the Ras signaling cascade by directly binding to Ras-GRF [31]. In the present study, we found that the activity of the Ras-NF-KB signaling pathway decreases after the Orai1 downregulation in MC3T3-E1 cells. These data suggest that the Orai1 downregulation can reduce the expression of cyclin D1 in osteoblasts by inactivating the Ras-NF-KB signaling pathway. To verify this result, the Ras signaling pathway inhibitor ARS-853 was used. The findings revealed that Orai1 siRNA did not further reduce the proliferation, the proportion of cells in the S phase, and the expression of cyclin D1 after the blockage of Ras signal transduction. These results confirm that the Orai1 downregulation reduces the proliferation of osteoblasts by inactivating the Ras-NF-KB signaling pathway.

\section{Conclusions}

Our study indicates that Orai1 downregulation leads to a slowdown of osteoblast proliferation and to cell cycle arrest. The Orai1 downregulation inactivates the Ras-NF-KB signaling pathway to reduce the 
expression of cyclin D1 thus decreasing the proliferation of osteoblasts. Abnormal Orai1 expression or function may be a major cause of osteoporosis development. Therefore, detailed research on the role of Orai1 in the onset of osteoporosis can yield a new idea for the biological treatment of osteoporosis.

\section{Abbreviations}

CRAC: calcium release-activated calcium, STIM1: stromal interaction molecule 1, ATCC: American Type Culture Collection, siRNA: small interfering RNA, PVDF: polyvinylidene fluoride microporous, ECL:

Enhanced Chemiluminescence, MTT: 3-(4,5-dimethylthiazol-2-yl)-2,5- diphenyltetrazolium bromide, Tunel: terminal deoxynucleotidyl transferase dUTP nick end labeling, DAPI: 4,6-diamidino-2-phenylindole, PI: propidium iodide, CDKs: cyclin-dependent kinases.

\section{Declarations}

Ethics approval and consent to participate: The study protocol was approved by the Ethics Committee of Hong Hui Hospital, Xi'an Jiao Tong University (No. 202103023). All procedures performed in studies were in accordance with the ethical standards of the institutional and/or national research committee. All authors have confirmed their participation in this study and agreed to publish this manuscript.

Consent for publication: Not applicable.

Availability of data and material: The datasets used and/or analysed during the current study are available from the corresponding author on reasonable request.

Competing interests: The authors declare that they have no financial and non-financial competing interests.

Funding: This study was supported by the Natural Science Foundation of Shannxi Province (2020JM687) and the Science and Technology Plan of Xi'an City (2020yb35).

Authors' contributions: GYS performed the experiments, analysed the data and wrote the manuscript; HDJ designed the study, supervised the study and revised the manuscript. All authors reviewed the manuscript.

Acknowledgements: We would like to thank Editage (www.editage.cn) for English language editing.

\section{References}

1. Phetfong J, Sanvoranart T, Nartprayut K, et al. Osteoporosis: the current status of mesenchymal stem cell-based therapy. Cell Mol Biol Lett. 2016;21:12.

2. Hu LF, Yin C, Zhao F, et al. Mesenchymal Stem Cells: Cell Fate Decision to osteoblasts or Adipocyte and Application in Osteoporosis Treatment. Int J Mol Sci. 2018;19(2): 360. 
3. Jiang ZW, Li Z, Zhang W, et al. Dietary Natural N-Acetyl-d-Glucosamine Prevents Bone Loss in Ovariectomized Rat Model of Postmenopausal Osteoporosis. Molecules. 2018;23(9): 2302.

4. Tarantino U, Iolascon G, Cianferotti $L$, et al. Clinical guidelines for the prevention and treatment of osteoporosis: summary statements and recommendations from the Italian Society for Orthopaedics and Traumatology. J Orthop Traumatol. 2017;18 Suppl 1:3-36.

5. Zhao B, Zhao WQ, Wang YQ, et al. Prior administration of vitamin K2 improves the therapeutic effects of zoledronic acid in ovariectomized rats by antagonizing zoledronic acid-induced inhibition of osteoblasts proliferation and mineralization. PLoS One. 2018;13(8): e0202269.

6. Nguyen NT, Han W, Cao WM, et al. Store-Operated Calcium Entry Mediated by ORAI and STIM. Compr Physiol. 2018;8(3):981-1002.

7. Cai XG, Robert M, Nwokonko NA. Pore properties of Orai1 calcium channel dimers and their activation by the Orai1 ER calcium sensor. J Biol Chem. 2018;293(33): 12962-12974.

8. Chang CL, Chen YJ, Quintanilla CG, et al. EB1 binding restricts Orai1 translocation to ER-PM junctions and regulates store-operated $\mathrm{Ca}^{2+}$ entry. J Cell Biol. 2018;217(6): 2047-2058.

9. Guo Y, Zhu J, Wang X, et al. Orai1 Promotes Osteosarcoma Metastasis by Activating the Ras-Rac1WAVE2 Signaling Pathway. Med Sci Monit. 2019;25: 9227-9236.

10. SY Hwang, JW Putney. Orai1-mediated calcium entry plays a critical role in osteoclast differentiation and function by regulating activation of the transcription factor NFATc1.Faseb Journal. 2012;26(4):1484-1492.

11. LJ Robinson, HC Blair, JB Barnett, J Soboloff.The roles of Orai and Stim in bone health and disease. Cell Calcium. 2019;81:51-58.

12. Choi H, Srikanth S, Atti E, et al. Deletion of Orai1 leads to bone loss aggravated with aging and impairs function of osteoblasts lineage cells. Bone Rep. 2018;8:147-155.

13. Cai XG, Robert M. Nwokonko, NA. Pore properties of Orai1 calcium channel dimers and their activation by the Orai1 ER calcium sensor. J Biol Chem. 2018;293(33): 12962-12974.

14. Peng S, Zhou G, Luk KD, et al. Strontium promotes osteogenic differentiation of mesenchymal stem cells through the CAMKII/MAPK signaling pathway. Cell Physiol Biochem. 2009;23(1-3):165-74.

15. Wajdner HE, Farrington J, Barnard C, et al. Orai and TRPC channel characterization in FceRI-mediated calcium signaling and mediator secretion in human mast cells. Physiol Rep. 2017; 5(5): e13166.

16. Staehlke S, Rebl H, Finke B, et al. Enhanced calcium ion mobilization in osteoblasts on amino group containing plasma polymer nanolayer. J. Barbara Nebe. Cell Biosci. 2018; 8: 22.

17. Wang H, Sun W, Ma JQ, et al. Polycystin-1 Mediates Mechanical Strain-Induced osteoblastsic Mechanoresponses via Potentiation of Intracellular Calcium and Akt/ $\beta$-Catenin Pathway. PLoS One. 2014;9(3): e91730.

18. Delaine-Smith RM, Sittichokechaiwut A, Reilly GC. Primary cilia respond to fluid shear stress and mediate flow-induced calcium deposition in osteoblasts. FASEB J. 2014;28(1): 430-439. 
19. Martineau X, Abed E, Martel-Pelletier J, et al..Alteration of Wnt5a expression and of the non-canonical Wnt/PCP and Wnt/PKC-Ca2 + pathways in human osteoarthritis osteoblasts. PLoS One. 2017;12(8): e0180711.

20. Cabral WA, Ishikawa M, Garten M, et al. Absence of the ER Cation Channel TMEM38B/TRIC-B Disrupts Intracellular Calcium Homeostasis and Dysregulates Collagen Synthesis in Recessive Osteogenesis Imperfecta. PLoS Genet. 2016;12(7): e1006156.

21. Peterlik M, Kállay E, Heide S. Calcium Nutrition and Extracellular Calcium Sensing: Relevance for the Pathogenesis of Osteoporosis, Cancer and Cardiovascular Diseases. Cross. Nutrients. 2013;5(1): 302-327.

22. Min P, L. Quarles D. osteoblasts Calcium-Sensing Receptor Has Characteristics of ANF/7TM Receptors. J Cell Biochem. 2005, 95(6): 1081-1092.

23. Tharmalingam S, David R. The Calcium-Sensing Receptor and Integrins in Cellular Differentiation and Migration.Hampson. Front Physiol. 2016;7:190.

24. Liu JR, Xu GM, Shi XS, et al. Low temperature plasma promoting fibroblast proliferation by activating the NF-KB pathway and increasing cyclinD1 expression. Sci Rep. 2017;7: 11698.

25. Casimiro MC, Sante GD, Ju XM et al. Cyclin D1 promotes androgen-dependent DNA damage repair in prostate cancer cells. Cancer Res. Cancer Res. 2016;76(2): 329-338.

26. Colleoni B, Paternot S, Pita JM, et al. JNKs function as CDK4-activating kinases by phosphorylating CDK4 and p21. Oncogene. 2017;36(30): 4349-4361.

27. Chen HB, Xu XW, Wang GP, et al. CDK4 protein is degraded by anaphase-promoting complex/cyclosome in mitosis and reaccumulates in early G1 phase to initiate a new cell cycle in HeLa cells. J Biol Chem. 2017;292(24): 10131-10141.

28. Papaioannou G, Mirzamohammadi F, Kobayashi T. Ras signaling regulates osteoprogenitor cell proliferation and bone formation. Cell death \& disease. 2016; 7 (10):e2405..

29. Hsieh CP, Chiou YL, Lin CY. Hyperbaric oxygen-stimulated proliferation and growth of osteoblasts may be mediated through the FGF-2/MEK/ERK 1/2/NF-KB and PKC/JNK pathways. Connect Tissue Res. 2010;51 (6): 497-509.

30. Granet C, Boutahar N, Vico L, Alexandre C, Lafage-Proust MH. MAPK and SRC-Kinases Control EGR-1 and NF-KB Inductions by Changes in Mechanical Environment in osteoblasts. Biochem Biophys Res Commun. 2001;284 (3): 622-31.

31. Keiper M, Stope MB, Szatkowski D, et al. Epac- and $\mathrm{Ca}^{2+}$-controlled activation of Ras and extracellular signal-regulated kinases by Gs-coupled receptors. J Biol Chem. 2004; 279(45):46497508.

\section{Figures}


a

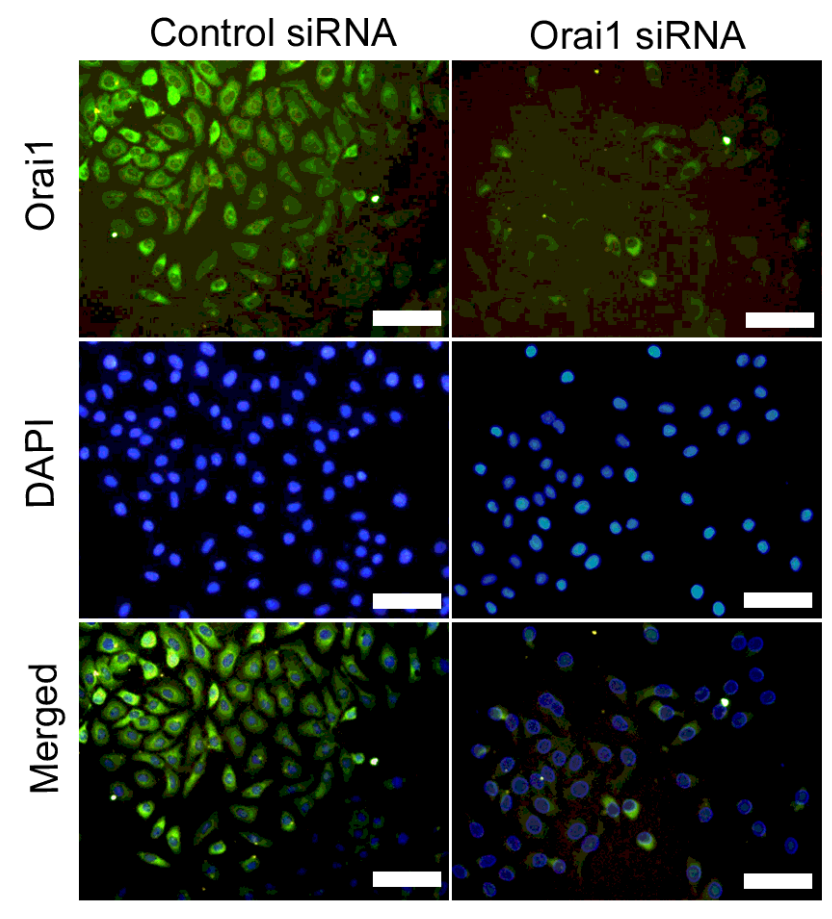

b

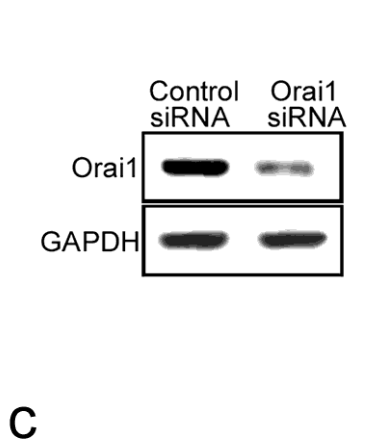

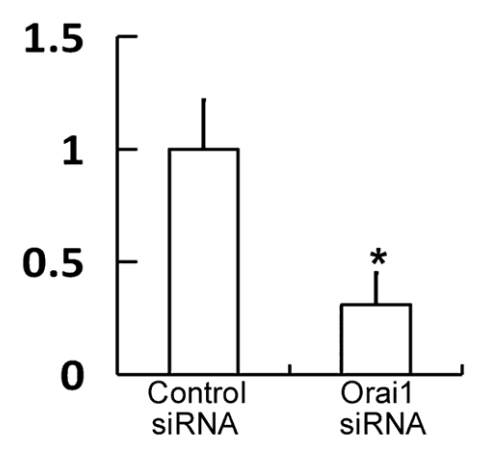

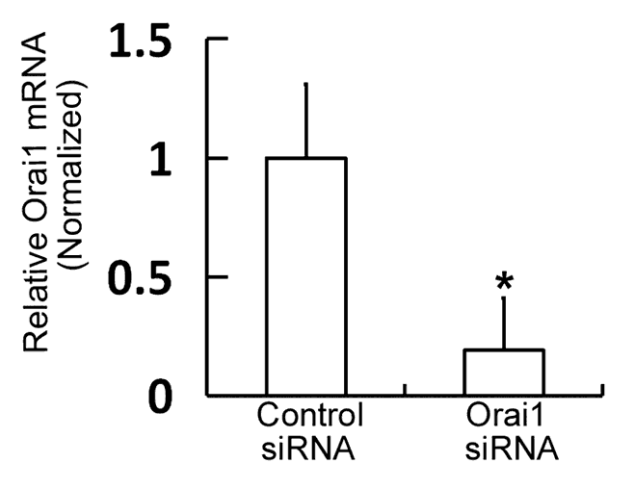

\section{Figure 1}

The expression of Orai1 is inhibited by Orai1 siRNA in MC3T3-E1 cells. (a) Orai1 protein levels were examined by immunofluorescence analysis in MC3T3-E1 cells transfected with either control siRNA or Orai1 siRNA (Scale $=100 \mu \mathrm{m}$ ). (b) Orai1 protein levels were examined by western blot analysis in MC3T3E1 cells transfected with either control siRNA or Orai1 siRNA. (c) The Orai1 mRNA levels were examined by real-time PCR in MC3T3-E1 cells transfected with either control siRNA or Orai1 siRNA. Data are presented as means \pm SD from three independent experiments. ${ }^{*} p<0.05$ as compared with the control.

\section{Figure 2}

Orai1 downregulation reduces the proliferation of MC3T3-E1 cells and causes their cell cycle arrest. (a) The cell proliferation was evaluated by the MTT assay of MC3T3-E1 cells transfected with either control siRNA or Orai1 siRNA. (b) Apoptosis was evaluated by Tunel staining of the MC3T3-E1 cells transfected with either control siRNA or Orai1 siRNA (Scale $=100 \mu \mathrm{m})$. (c) The cell cycle distribution was investigated using PI staining of the MC3T3-E1 cells transfected with either control siRNA or Orai1 siRNA. Data are presented as means \pm SD from three independent experiments. ${ }^{*} p<0.05$ as compared with the control. 
a
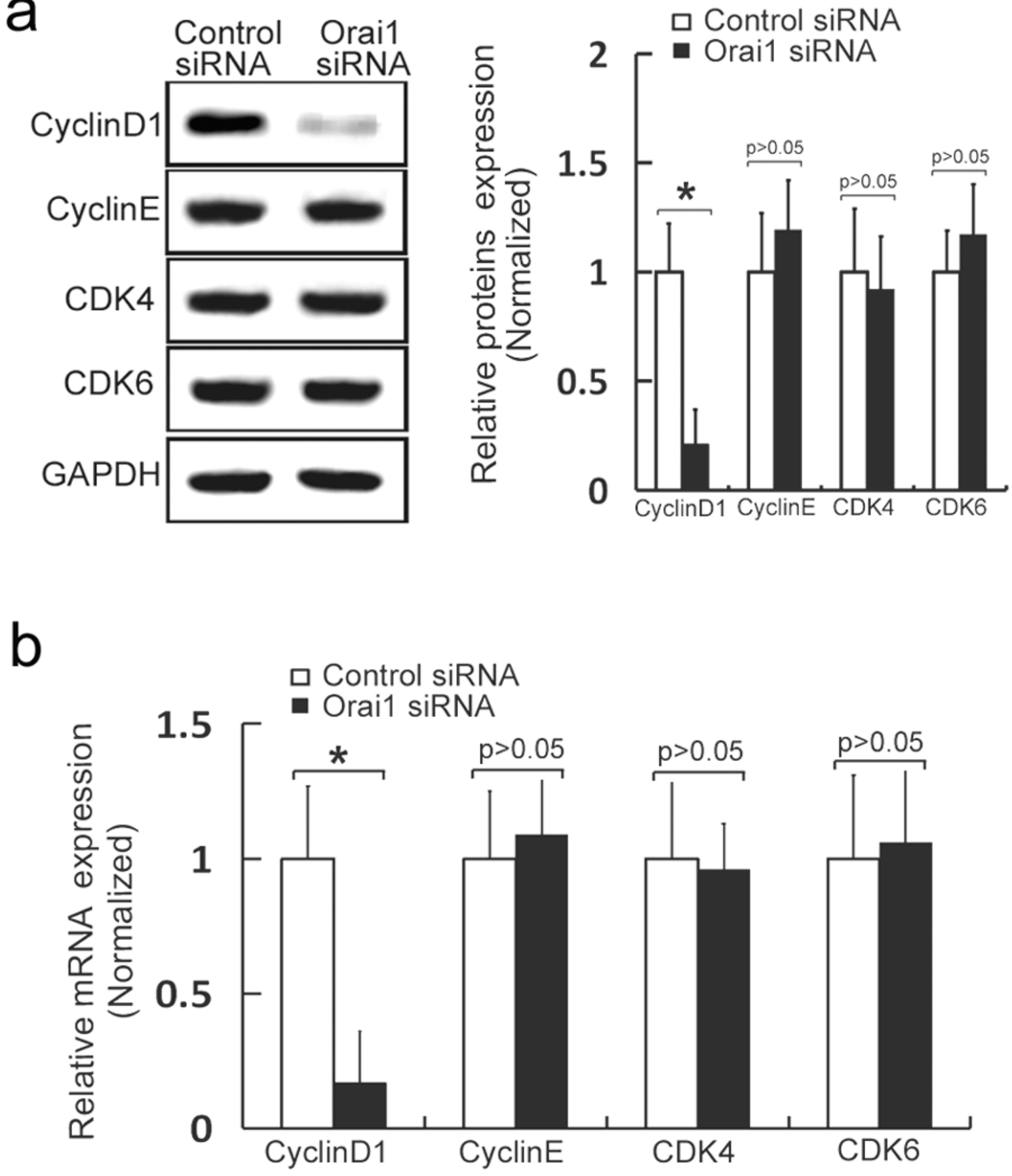

Figure 3

Orai1 downregulation lowers the expression of cyclin D1 in MC3T3-E1 cells. (a) Western blotting was performed to assess the expression levels of cyclin D1, cyclin E, CDK4, and CDK6 in the MC3T3-E1 cells transfected with either control siRNA or Orai1 siRNA. (b) Real-time PCR was carried out to measure mRNA levels of cyclin D1, cyclin E, CDK4, and CDK6 in the MC3T3-E1 cells transfected with either control siRNA 
or Orai1 siRNA. Data are presented as means \pm SD from three independent experiments. ${ }^{*} p<0.05$ as compared with the control.

\section{Figure 4}

Orai1 downregulation reduces the proliferation of osteoblasts by inactivating the Ras-NF-KB signaling pathway. (a) The amounts of Ras-GRF and p-p65-NF-kB were measured in the MC3T3-E1 cells transfected with either control siRNA or Orai1 siRNA. ARS-853 was dissolved in DMSO to prepare a stock solution. After MC3T3-E1 cells were transfected with either the control siRNA or Orai1 siRNA, the ARS-853 stock solution was diluted to a concentration of $50 \mathrm{nM}$ in the cell culture medium. DMSO alone was dissolved as negative (vehicle) control. After that, western blotting and cell proliferation and cell cycle assays were performed after incubation with or without the Ras signaling pathway inhibitor ARS-853 (50 $\mathrm{nM}$ ) for an appropriate period. (b) After incubation with or without ARS-853, the amounts of p-p65-NF-KB and cyclin D1 were evaluated in the MC3T3-E1 cells transfected with either the control siRNA or Orai1 siRNA. (c) After incubation with or without ARS-853, the proliferation rate of the MC3T3-E1 cells (transfected with either the control siRNA or Orai1 siRNA) was evaluated. (d) After incubation with or without ARS-853, the cell cycle distribution was investigated for the MC3T3-E1 cells transfected with either the control siRNA or Orai1 siRNA. Data are presented as means \pm SD from three independent experiments. ${ }^{*} \mathrm{p}<0.05$ as compared with the control. 